\title{
Mortalidad excepcional en los cricétidos (Rodentia) del Mioceno medio de Somosaguas (Pozuelo de Alarcón, Madrid)
}

\section{Exceptional mortality model in cricetids (Rodentia) of the middle Miocene from Somosaguas (Pozuelo de Alarcón, Madrid)}

\author{
I. Menéndez $z^{1,2}$, A.R. Gómez Cano ${ }^{3,4}$, M. Hernández Fernández ${ }^{1,2}$ \\ 1 Departamento de Paleontología, Facultad de Ciencias Geológicas, Universidad Complutense de Madrid, José Antonio \\ Novais 2, Madrid 28040, Spain. Email: irismene@ucm.es. ORCID ID: http://orcid.org/0000-0003-1866-8351, http://orcid. \\ org/0000-0001-5640-9647 \\ 2 Departamento de Cambio Medioambiental, Instituto de Geociencias (UCM, CSIC), José Antonio Novais 2, Madrid 28040, Spain. \\ 3 Transmitting Science, C/Gardenia 2, 08784 Piera, Barcelona (Spain). Email: argomezcano@gmail.com. ORCID ID: http:// \\ orcid.org/0000-0001-5351-4185 \\ 4 Institut Català de Paleontologia Miquel Crusafont, Universitat Autónoma de Barcelona. Carrer de les Columnes s/n, Campus \\ de la UAB, 08193 Cerdanyola del Vallès, Barcelona (Spain).
}

\section{RESUMEN}

En este estudio se analizó el espectro de desgaste dental de los molares, superiores e inferiores, de individuos de las dos especies de roedores más abundantes registradas en el yacimiento de Somosaguas Sur: Megacricetodon collongensis (MEIN, 1958) y Democricetodon larteti (SCHAUB, 1925). Los resultados muestran gran abundancia de molares poco desgastados en ambas especies, lo que indica la existencia de una mortalidad fuertemente sesgada hacia los individuos juveniles. Este modelo de mortalidad refleja un suceso catastrófico de larga duración y se asocia a periodos de sequía prolongados que produjeron la muerte temprana de los individuos durante generaciones. Esto es coherente con los datos obtenidos para los macromamíferos de Somosaguas donde también existe una mortalidad preferencial de los individuos juveniles asociada a eventos de gran aridez. A pesar de que todos los molares mostraron el mismo patrón de mortalidad preferencial en individuos jóvenes, ambas especies presentaron diferentes niveles de desgaste entre los distintos molares, lo cual sugiere que el proceso de masticación pudo ser distinto entre ambas especies o que el inicio del desgaste de los molares anteriores pudo iniciarse durante la lactancia. Finalmente, el desgaste diferencial observado entre los $\mathrm{M} 1 \mathrm{de} M$. collongensis y $D$. larteti podría deberse a la distinta morfología de las cúspides y valles de sus molares, lo que provocaría que el desgaste no se produjese de la misma manera en las dos especies, o a ligeras diferencias dietarias.

Palabras clave: Cricetidae; desgaste dental; Morfología; mortandad; Aragoniense dx.doi.org/10.3989/egeol.42347.397.

Copyright: (c) 2017 CSIC. This is an open-access article distributed under the terms of the Creative Commons Attribution-Non Commercial (by-nc) Spain 3.0 License. 


\section{ABSTRACT}

In this work we studied the wear degree of the superior and lower molars of the two most abundant rodent species from the South Somosaguas fossil site: Megacricetodon collongensis (MEIN, 1958) and Democricetodon larteti (ScHAUB, 1925). The results show a great abundance of elements with scarce tooth wear in both species, which indicates the existence of a high mortality of young individuals. This mortality model shows an enduring catastrophic event associated to long periods of aridity that produced juvenile mortality along generations. This tendency of mortality fits in the patterns obtained from other works from Somosaguas where the existence of high rates of deaths of young individuals is associated with events of high aridity. $D$. larteti and $M$. collongensis have different patterns of wear degree along their dental rows, which could indicate that the mastication process was different in both species or maybe that the tooth wear started earlier. Finally, the differences in wear degree observed in the M1 of $M$. collongensis and $D$. larteti can be explained by the different morphology of the peaks and valleys of the molars, which cause a faster wear of the first molar of $D$. larteti than in those of $M$. collongensis; or by light dietary differences.

Keywords: Cricetidae; dental wear; Morphology; mortality; Aragonian

\section{Introducción}

Desde Kurtén (1953), los estudios paleontológicos proponen dos modelos de mortalidad principales que describen procesos diferenciales de formación del registro (Fig. 1). De manera general, el perfil atricional muestra una sobrerrepresentación de individuos muy jóvenes y muy viejos, mientras que los adultos están subrepresentados en relación a sus frecuencias en la población viva. Este tipo de mortalidad selectiva se debe a la susceptibilidad diferencial de las distintas categorías de edad a diferentes factores de mortandad (ej. predación, enfermedad, escasez de recursos, etc.). Por otro lado, el perfil catastrófico exhibe una distribución de frecuencias que disminuye gradualmente conforme aumenta la edad de la muerte, lo cual refleja las abundancias relativas de la población en vida (Lyman 1994; Domingo, M.S. et al., 2012). No obstante, existen numerosos factores interrelacionados que pueden dar lugar a que ambos patrones se vean modificados (Klein, 1982; Lyman, 1994).

Sin embargo, en el caso del Mioceno medio de Somosaguas se ha argumentado la existencia de una mortalidad preferencial exclusiva de individuos juveniles, tanto en macromamíferos (López Martínez et al., 2000; Polonio \& López Martínez, 2000; Manzanero \& Hernández Fernández, 2014) como en roedores (Torroba et al., 2010). Este patrón ha sido asociado a eventos de aridez extrema en un marco macroclimático tropical semiárido (Hernández Fernández et al., 2006; Domingo, L. et al., 2012; García Yelo et al., 2014; Domingo et al., 2017). Estas condiciones paleoclimáticas fueron inferidas a partir de análisis faunísticos (Hernández Fernández et al., 2003, 2006; Perales et al., 2009), así como distintos análisis geológicos del yacimiento, tanto sedimentológicos y mineralógicos (Carrasco et al., 2008; Fesharaki et al., 2015) como geoquímicos (Domingo et al., 2009). Según esta hipótesis, sequías prolongadas producirían un incremento de la mortandad, que afectaría en particular a los individuos juveniles, dando lugar a una acumulación diferencial de los restos en el área del yacimiento en épocas de lluvia (Polonio \& López Martínez, 2000; Hernández Fernández et al., 2006; Martín Perea, 2015; Domingo et al., en prensa).
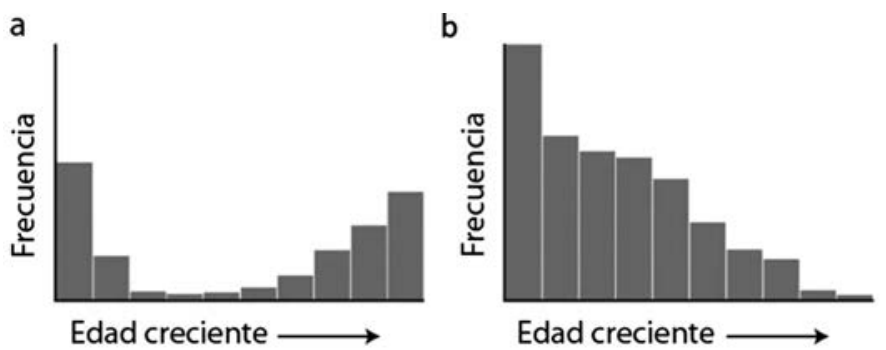

Fig. 1.-Perfiles clásicos de mortalidad. A) Patrón atricional (en forma de U). En este perfil los miembros más débiles de la población, que son los más jóvenes y, en menor medida, los más viejos, están sobrerrepresentados en relación a sus frecuencias en la población viva. B) Patrón catastrófico (en forma de L). Este perfil reproduce la distribución de edades de la población en vida. Cada barra corresponde a una categoría de edad. Modificado de Domingo, M.S. et al. (2012). 
Uno de los objetivos de este estudio es comprobar esta hipótesis a partir del análisis de una de las especies más abundantes del yacimiento, el cricétido Megacricetodon collongensis (MEIN, 1958). Si la mortandad de esta especie también estuvo regulada por eventos climáticos extremos, ésta debería resultar equivalente a la observada con anterioridad en otras especies del yacimiento. Para comprobarlo se comparó con el roedor Democricetodon larteti (SCHAUB, 1925), que ya fue utilizado en trabajos anteriores en los que se verificó una mortalidad preferencial de individuos juveniles de esta especie en el yacimiento de Somosaguas a partir del estudio del desgaste de los terceros molares (Torroba et al., 2010).

Como objetivo adicional, se analizó el desarrollo del desgaste de cada molar para establecer si todos se comportan de la misma forma. Si así fuera, todas estas piezas dentales podrían utilizarse indistintamente para estudios de mortalidad. Para ello se estudiaron las diferencias intraespecíficas en el desgaste de cada pieza yugal estudiada por separado. Por último, se estudiaron las diferencias interespecíficas comparando la media del desgaste de cada molar de $M$. collongensis con su relativo de $D$. larteti. De no encontrarse diferencias entre ambas especies, podría confirmarse una influencia preponderante de los factores externos en el desarrollo del patrón de mortalidad.

\section{Material y métodos}

\section{Área y material de estudio}

El yacimiento paleontológico estudiado está situado en Pozuelo de Alarcón, al oeste de la ciudad de Madrid (España), dentro del campus que la Universidad Complutense de Madrid tiene junto a la localidad de Somosaguas. Luis \& Hernando (2000) incluyeron el yacimiento en la biozona E del Aragoniense medio (Mioceno medio) definida en la cuenca de CalatayudDaroca (Daams \& Freudenthal, 1981, 1988; Daams et al., 1999; van der Meulen et al., 2012) y datada en alrededor de $14 \mathrm{Ma}$ (van der Meulen et al., 2011; Hernández-Ballarín et al., 2011).

Se han realizado excavaciones sistemáticas desde 1998 y se han reconocido dos yacimientos: Somosaguas Norte, donde se registran principalmente macromamíferos, y Somosaguas Sur, donde se registran restos de micromamíferos. En ambos yacimientos se han distinguido tres tramos sedimentarios (T1, T2 y T3) que corresponden a depósitos terrígenos de abanicos aluviales procedentes del Sistema Central (Mínguez Gandú, 2000), dentro de una secuencia de alternancia entre arcillas y arcosas (Díez-Canseco et al., 2012).

La asociación de vertebrados de Somosaguas es una de las más completas del Mioceno medio de la cuenca de Madrid, reuniendo entre los dos yacimientos (Somosaguas Norte y Sur) un total de 29 especies de vertebrados, 24 de las cuales pertenecen a mamíferos (Hernández Fernández et al., 2006; Perales et al., 2009). Los análisis paleoambientales sugieren que durante el Mioceno en Somosaguas se extendía una sabana tropical o subtropical rodeando un lago temporal, en un contexto paleoclimático global cada vez más fresco y árido como consecuencia del restablecimiento de la capa de hielo de la Antártida (López-Martínez et al., 2000; Hernández Fernández et al., 2006; Carrasco et al., 2008; Domingo et al., 2009; Perales et al., 2009; García Yelo et al., 2014; Fesharaki et al., 2015).

Los restos dentales de los micromamíferos resultan piezas extremadamente importantes en los estudios paleontológicos, dado que se trata de las piezas más mineralizadas y, por tanto, están ampliamente representadas en el registro fósil. Además son las piezas diagnósticas para la sistemática de los distintos grupos (e.g. Freudenthal, 1966; Álvarez Sierra et al., 1987; van der Meulen et al., 2003). En conjunto, un $52 \%$ del total de restos catalogados e inventariados en Somosaguas son restos de micromamíferos identificados a nivel de especie. Durante los últimos 18 años se ha extraído sedimento con contenido fósil del tramo T1 en Somosaguas-Sur (Fesharaki et al., 2012), el cual ha sido lavado-tamizado y posteriormente triado (Daams \& Freudenthal, 1988; López Martínez, 1992; Luis \& Hernando, 2000). En este trabajo se estudió el material dental de las dos especies de cricétidos más abundantes procedentes de Somosaguas Sur, estudiado sistemáticamente por Luis \& Hernando (2000). Estos autores describieron la presencia de Megacricetodon collongensis y Falbuschia darocensis. Posteriormente, la revisión del material ibérico de este último género (van der Meulen et al., 2003) ha dado lugar a la reasignación del material de Somosaguas a Democriceton larteti. Estas dos especies suponen cerca del 80\% 
del total de la asociación de roedores registrada en el yacimiento (Hernández Fernández et al., 2006; Blanco \& Hernández Fernández, 2016).

Estas especies presentan tres molares superiores (M1, M2 y M3) y tres inferiores $(\mathrm{m} 1, \mathrm{~m} 2$ y $\mathrm{m} 3)$. Sus molares se caracterizan por un patrón dental conservador de tipo braquiodonto y bunolofodonto, distinguiéndose Democricetodon larteti de Megacricetodon collongensis por el mayor tamaño de su dentición, así como por la mayor voluminosidad de sus cúspides.

Se estudiaron únicamente dientes completos y sin fragmentación aparente en la superficie oclusal (Megacricetodon, $\mathrm{N}=251$; Democricetodon, $\left.\mathrm{N}=141 ; \mathrm{N}_{\text {total }}=392\right)$. El material estudiado se encuentra depositado en la colección del Departamento de Paleontología de la Universidad Complutense de Madrid.

\section{Análisis de mortandad}

Existen diversos métodos para estimar la edad con la que murieron los individuos registrados en un yacimiento (Morris, 1972). Algunas técnicas se basan en el estudio de los cambios producidos por el crecimiento del estado inmaduro al adulto en huesos particulares en partes específicas de un hueso (Krogman \& İşcan, 1986; Ubelaker, 1987; White, 1991). No obstante, debido a los elevados niveles de fragmentación de los fósiles de Somosaguas y, en particular, a que los restos de roedores suelen estar dominados por piezas dentales aisladas, no podemos utilizar aquellos métodos que se basan en parámetros derivados de restos óseos completos. Por otro lado, se pueden inferir las edades relativas de muerte analizando el desarrollo y la erupción de las piezas dentales (Domingo et al. 2012), contabilizando las capas crecimiento anual de dentina (Laws, 1952; Hohn et al. 1989), o mediante el desgaste dental (Freudenthal et al., 2002; Galbany et al., 2014).

Para estimar la mortalidad a partir de dentición aislada utilizamos la metodología propuesta por Freudenthal et al. (2002), que estudia el desgaste de los terceros molares asumiendo que cuanto más desgastados resulten los mismos, mayor será la edad del individuo. Se utilizó este método continuando la línea de trabajos anteriores sobre mortalidad de micromamíferos del yacimiento de Somosaguas Sur en los que se aplicó esta misma metodología (Torroba et al., 2010; Menéndez et al., 2015). No obstante, al estudiar tan sólo los terceros molares existe el riesgo de sesgo de muestreo asociado al pequeño tamaño de estas piezas dentales, en particular en aquellas especies de menor porte, el cual posiblemente les permita atravesar la luz de malla de los tamices utilizados para lavar el sedimento (0,7 mm) (Menéndez et al., 2015). Por ello, con la finalidad de elaborar espectros de desgaste más fiables, además de los terceros molares, se estudiaron también los primeros y los segundos molares.

El método desarrollado por Freudenthal et al. (2002) se basa en el índice de desgaste de los molares - Wear Index, WI $=100 \mathrm{D} /(\mathrm{D}+\mathrm{E})-$, expresado como el porcentaje de la superficie oclusal ocupada por dentina (D) en comparación con la superficie total (Fig. 2), derivada de la suma de la superficie ocupada por dentina y por esmalte $(\mathrm{D}+\mathrm{E})$. Este índice será mayor cuanto más desgastadas estén las piezas dentales en estudio, relacionando un mayor desgaste del molar con una mayor edad del individuo.

Utilizando una cámara Nikon D300s, equipada con una lente Nikon AF-S VR 105 mm f/2.8 IF-ED y tres anillos de extensión, se fotografiaron todos los molares superiores e inferiores a estudiar de ambas especies. Para calcular el índice de desgaste (WI) se midieron la proyección de la superficie expuesta de dentina y la proyección de superficie oclusal total observadas en las fotografías mediante Adobe Photoshop CS4.

Considerando que los individuos más jóvenes serían los que tienen un índice de desgaste menor, mientras que los individuos de mayor edad tendrían mayor porcentaje de diente desgastado (Freudenthal et al. 2002; Torroba et al. 2010), dividimos la distribución del índice de desgaste en diez categorías en función del porcentaje de superficie desgastada $(0-10 ; 10,1-20 ; 20,1-30 ; 30,1-40 ; 40,1-50 ; 50,1-60$; $60,1-70 ; 70,1-80 ; 80,1-90$ y 90,1-100\%). Esto permitió resumir los datos en histogramas porcentuales que representan la proporción de molares que se han encontrado para cada categoría de desgaste. Denominamos espectros de desgaste a estos histogramas, los cuales permiten inferir la mortalidad 


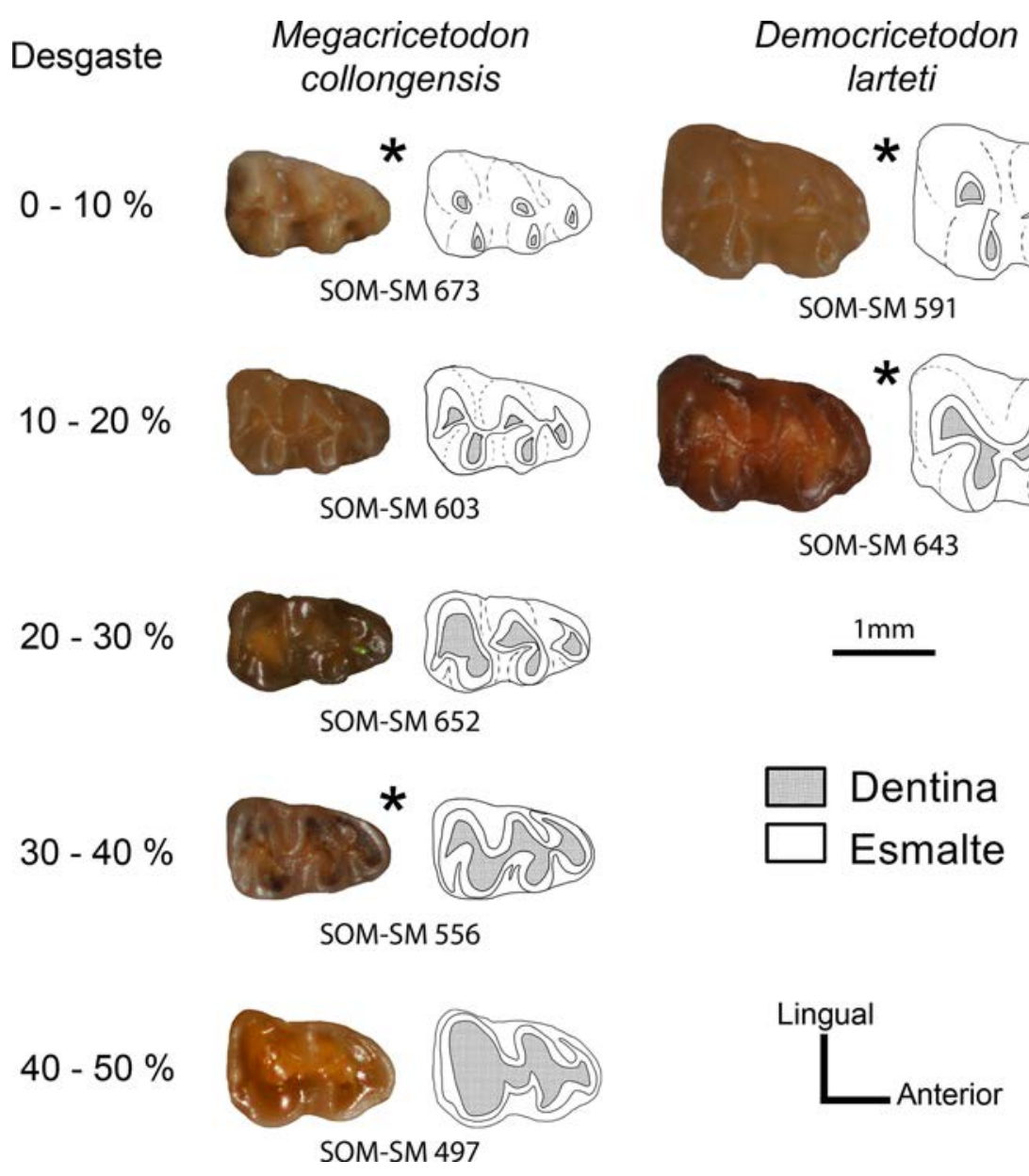

Fig. 2.-Vistas oclusales de varios $\mathrm{m} 1$ de Megacricetodon collongensis y Democricetodon larteti, correspondientes a las categorías de desgaste registradas. Se indican las superficies ocupadas por dentina y por esmalte. Se presentan m1 derechos, salvo los marcados con un * que representan imágenes especulares de $\mathrm{m} 1$ izquierdos.

de las especies estudiadas: si la mayor parte de los molares registrados en el yacimiento están en categorías poco desgastadas se atribuye a una mayor mortalidad de individuos juveniles; sin embargo, si se encuentran molares muy desgastados se infiere que los individuos eran ya adultos en el momento de la muerte.

\section{Análisis estadístico}

Los espectros de desgaste de ambas especies se examinaron a través de un análisis de comparación de histogramas porcentuales por medio del coeficiente de correlación no paramétrica de Spearman $(\rho)$, utilizando el programa SPSS v. 15.0 (SPSS, 2006).
Acorde con la hipótesis inicial sobre la incidencia de los periodos extremos de aridez en las poblaciones de los cricétidos del Mioceno de Somosaguas, se espera que ambas especies muestren una mortalidad preferencial de individuos juveniles. Por lo tanto se espera que los espectros de desgaste obtenidos para $M$. collongensis y $D$. larteti sean similares y que los resultados de las correlaciones no paramétricas de Spearman $(\rho)$ resulten significativos. Además, se realizó una prueba no paramétrica Kruskal-Wallis de comparación de distribuciones para determinar las diferencias en el desgaste de entre los distintos molares de cada especie. Finalmente, con el objetivo de determinar la significación estadística en las diferencias de desgaste entre ambas especies, para cada molar 
se realizó una prueba no paramétrica U de MannWhitney comparando la distribución de los datos de desgaste obtenida en cada especie.

\section{Resultados y discusión}

\section{Tendencias de mortalidad}

Una vez establecidos los espectros de desgaste para cada molar, superior o inferior, de cada especie (Figs. 3 y 4) se puede observar la ausencia de dientes entre las categorías más desgastadas (desde el $60 \%$ al $100 \%$ ) de las dos especies. En el total de la muestra correspondiente a las dos especies analizadas tan solo 13 dientes se encontraron entre el $40 \%$ y el $60 \%$ de desgaste, mientras que los
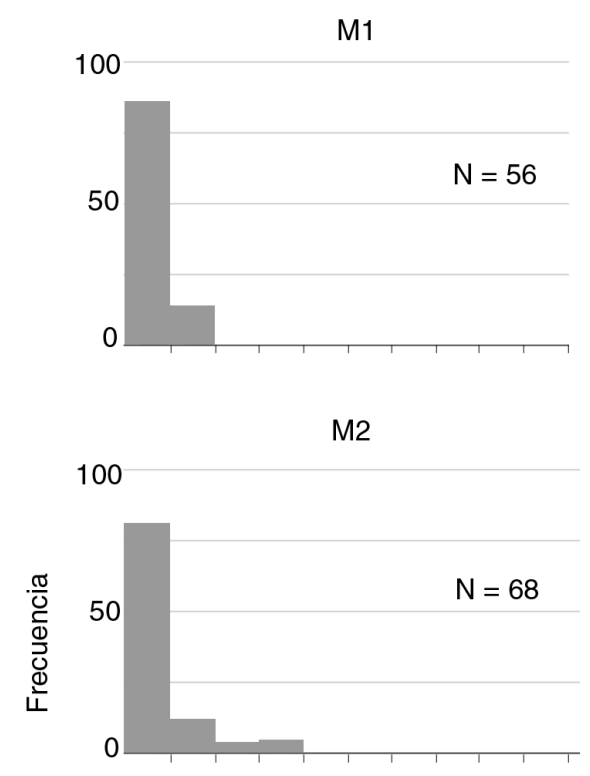

M3

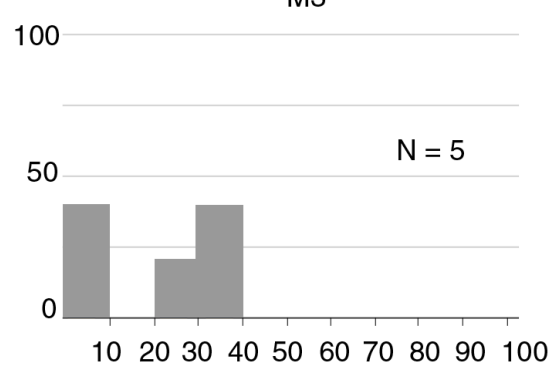

379 molares restantes presentaron un desgaste por debajo del $40 \%$. Esto parece indicar una clara preponderancia de individuos relativamente jóvenes en la asociación (Figs. 3 y 4).

Los espectros de desgaste obtenidos para cada molar de M. collongensis correlacionaron positivamente con los correspondientes de $D$. larteti, a excepción de los patrones obtenidos entre los M3, donde no se encontró una correlación significativa $(\rho=0,488 ; p=0,152 ;$ Tabla 1$)$.

Del mismo modo, el espectro de desgaste obtenido del M3 de M. collongensis es el único que mostró correlaciones no significativas al compararse con los espectros de desgaste del resto de molares de su misma especie, particularmente con M1, m2 y m3 (Tabla 1), el cual a su vez tampoco se correlacionó

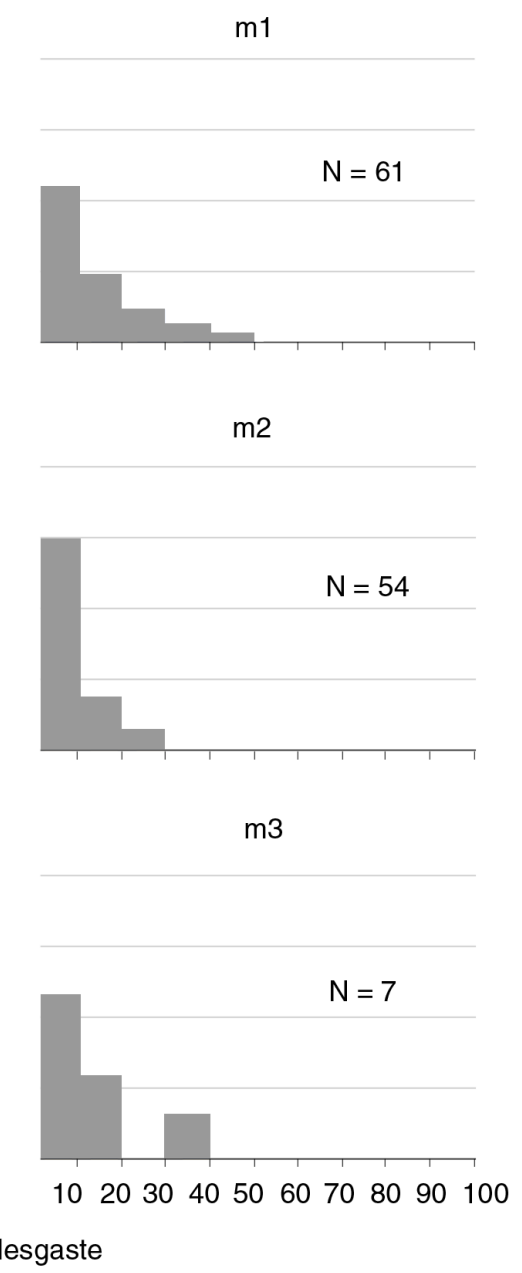

Fig. 3.-Espectros de desgaste de los molares superiores e inferiores de Megacricetodon collongensis en Somosaguas Sur. En el eje $\mathrm{X}$ se agrupan en clases los especímenes en base al porcentaje de desgaste. En el eje Y se representa las frecuencias porcentuales en cada categoría. 

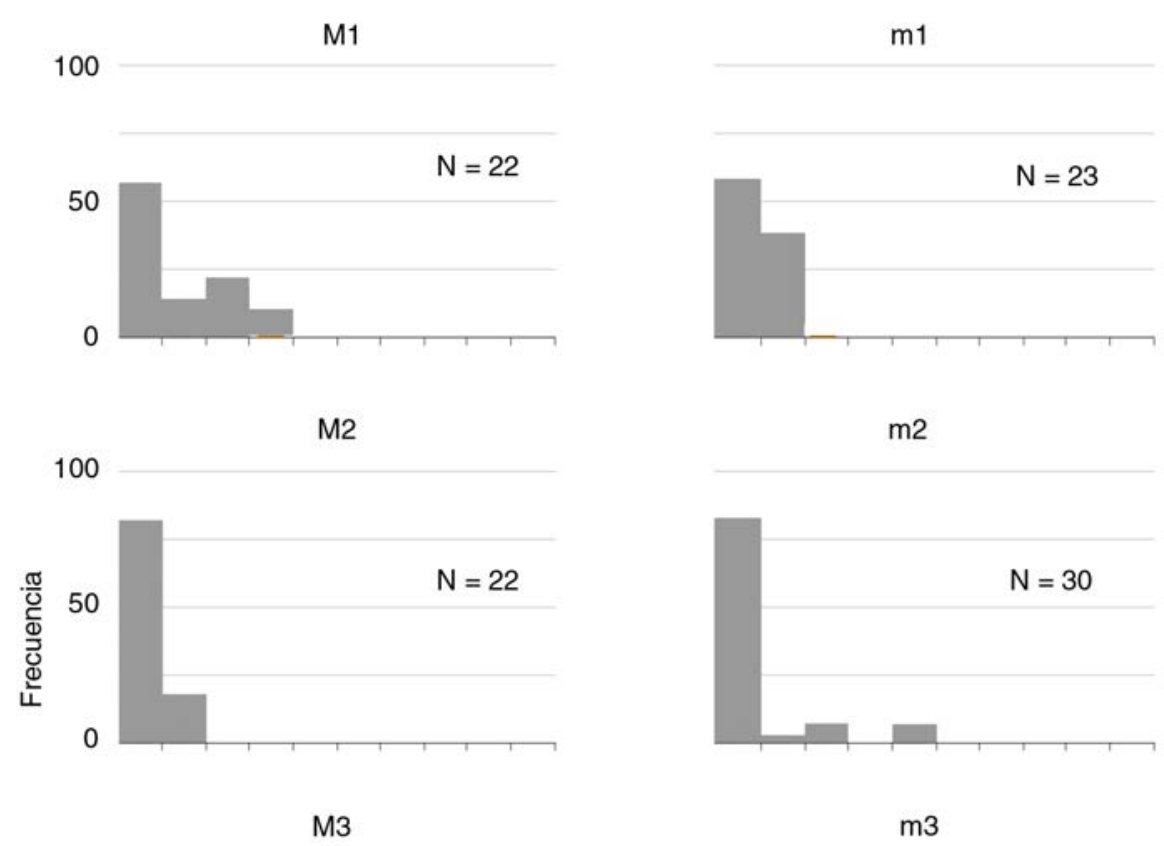

100
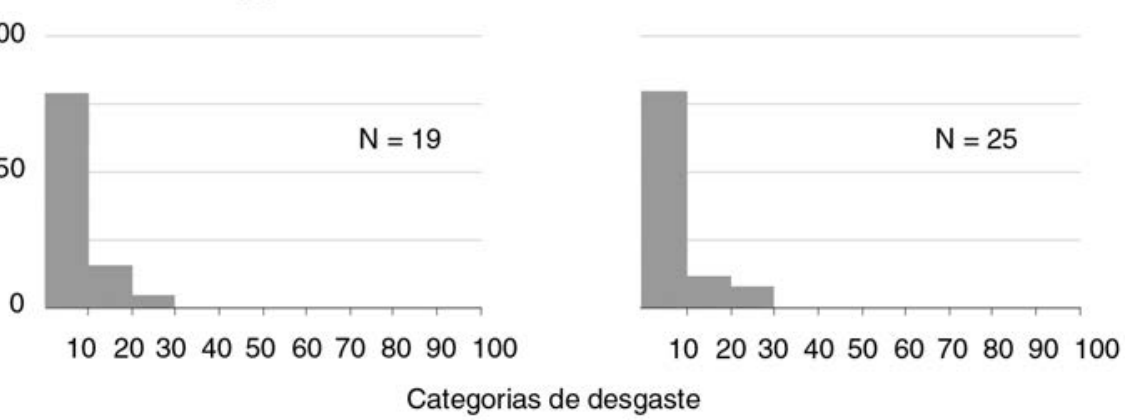

Fig. 4.-Espectros de desgaste de los molares superiores e inferiores de Democricetodon larteti en Somosaguas Sur. En el eje X se agrupan en clases los especímenes en base al porcentaje de desgaste. En el eje $Y$ se representa las frecuencias porcentuales en cada categoría.

con M2 y m1 más que de una manera marginalmente significativa. En el caso de D. larteti todas las piezas dentales mostraron patrones de desgaste congruentes, aunque en el caso de la correlación entre los espectros de desgaste de $\mathrm{m} 2$ con M2 y $\mathrm{m} 1$ tan sólo se encontró una correlación marginalmente significativa (Tabla 1 ).

A partir de estos resultados se puede inferir que el espectro de desgaste dental de $M$. collongensis y $D$. larteti fue muy similar, concordando también con los resultados en otros mamíferos del yacimiento de Somosaguas en los que se observó una mortalidad preferencial de individuos juveniles (Polonio \& López-Martínez, 2000; Manzanero \& Hernández Fernández, 2014), aunque el número de restos recuperados para las especies de macromamíferos no es suficiente para poder abordar un análisis estadístico comparativo con los resultados obtenidos aquí. Esta mortalidad preferencial de individuos muy jóvenes pudo ser provocada por un clima con una aridez muy marcada y periodos de sequía extrema (LópezMartínez et al., 2000).

La distribución de mortandad entre las categorías de edad no se amolda a un patrón atricional, que reflejaría la muerte preferencial de los individuos más débiles: muy jóvenes y viejos. Sin embargo, se adapta a un modelo catastrófico (con forma de L) con una particularidad. Mientras que en un modelo catastrófico se ven representadas todas las categorías de edad, en Somosaguas se observan tan solo restos de las categorías más jóvenes. Esto puede deberse a que los individuos estaban sometidos a una gran 
Tabla 1.-Análisis de comparación de histogramas porcentuales por medio del coeficiente de correlación no paramétrica de Spearman $(\rho)$. En fondo gris los datos obtenidos al comparar cada pieza dental de manera interespecífica. En la parte superior derecha se muestran los resultados al comparar cada pieza dental de forma intraespecífica de Megacricetodon collongensis y en la parte inferior izquierda de Democricetodon larteti.

\begin{tabular}{|c|c|c|c|c|c|c|c|c|c|c|}
\hline & & & \multicolumn{6}{|c|}{ Megacricetodon collongensis } & & \\
\hline & & & M1 & M2 & M3 & $\mathrm{m} 1$ & m2 & m3 & & \\
\hline \multirow{14}{*}{$\begin{array}{l}\text { Democricetodon } \\
\text { larteti }\end{array}$} & M1 & $\rho$ & 0,702 & 0,789 & 0,326 & 0,747 & 0,862 & 0,745 & \multirow{2}{*}{ M1 } & \multirow{13}{*}{$\begin{array}{r}\text { Megacricetodon } \\
\text { collongensis }\end{array}$} \\
\hline & & $\mathrm{p}$ & 0,024 & 0,007 & 0,358 & 0,013 & 0,001 & 0,013 & & \\
\hline & M2 & $\rho$ & 0,702 & 0,789 & 0,751 & 0,932 & 0,848 & 0,588 & \multirow{2}{*}{ M2 } & \\
\hline & & $\mathrm{p}$ & 0,024 & 0,007 & 0,012 & $<0,001$ & 0,002 & 0,074 & & \\
\hline & M3 & $\rho$ & 0,899 & 0,862 & 0,488 & 0,687 & 0,488 & 0,574 & \multirow{2}{*}{ M3 } & \\
\hline & & $\mathrm{p}$ & $<0,001$ & 0,001 & 0,152 & 0,028 & 0,152 & 0,083 & & \\
\hline & $\mathrm{m} 1$ & $\rho$ & 0,702 & 1,000 & 0,862 & 0,747 & 0,867 & 0,557 & \multirow{2}{*}{$\mathrm{m} 1$} & \\
\hline & & $\mathrm{p}$ & 0,024 & $<0,001$ & 0,001 & 0,013 & 0,001 & 0,094 & & \\
\hline & m2 & $\rho$ & 0,703 & 0,616 & 0,784 & 0,616 & 0,784 & 0,643 & \multirow{2}{*}{ m2 } & \\
\hline & & $\mathrm{p}$ & 0,023 & 0,058 & 0,007 & 0,058 & 0,007 & 0,045 & & \\
\hline & \multirow[t]{3}{*}{ m3 } & $\rho$ & 0,899 & 0,862 & 1,000 & 0,862 & 0,784 & 0,643 & \multirow{3}{*}{ m3 } & \\
\hline & & $\mathrm{p}$ & $<0,001$ & 0,001 & $<0,001$ & 0,001 & 0,007 & 0,045 & & \\
\hline & & & M1 & M2 & M3 & $\mathrm{m} 1$ & m2 & m3 & & \\
\hline & \multicolumn{9}{|c|}{ Democricetodon larteti } & \\
\hline
\end{tabular}

presión de supervivencia, por lo que nunca llegaban a alcanzar la senectud. Esta presión que afecta a la supervivencia estaría producida por periodos de sequía prolongada, de manera que el suceso catastrófico fue de larga duración. Este patrón se ajusta a lo que Menéndez et al. (2016) han descrito como un patrón espartano.

Estudios realizados sobre poblaciones actuales también presentan una distribución de categorías de desgaste significativamente diferente de la observada en los cricétidos de Somosaguas, no observándose esa ausencia de individuos de las categorías de edad más avanzada (Freudenthal et al., 2002; Torroba et al., 2010).

Tan sólo discrepan los datos obtenidos sobre el M3, que no presentan el mismo espectro de desgaste en una y otra especie. Esta discrepancia se debe a que existe un sesgo en el muestreo de los terceros molares de $M$. collongensis (Menéndez et al., 2015). El tamaño muestral de este estudio es de un total de 392 molares, 251 de ellos pertenecientes a $M$. collongensis, entre los cuales tan sólo 12 eran terceros molares ( $5 \mathrm{M} 3$ y $7 \mathrm{~m} 3$ ), mientras que en el caso de $D$. larteti, los terceros molares constituyen más del 30 $\%$ de los molares de esta especie (19 M3 y 25 m3).
Además, el espectro obtenido de los M3 de M. collongensis también se diferencia significativamente del M1, m2 y m3 de la misma especie. Este sesgo de muestreo en $M$. collongensis está probablemente ligado al pequeño tamaño de los terceros molares de esta especie, significativamente más pequeños que los de D. larteti (Luis \& Hernando, 2000).

Por lo tanto, los resultados que el análisis ofrece para el caso de los M3 de M. collongensis deben tomarse con precaución ya que, debido a lo anteriormente expuesto, la muestra puede carecer de individuos suficientes como para ser representativa de la asociación.

\section{Incidencia de desgaste intraespecífica}

Con respecto al grado de similitud intraespecífica en el espectro de desgaste, la media del porcentaje de desgaste es similar entre los distintos dientes de $M$. collongensis, pero no entre los de $D$. larteti (Fig. 5). Los resultados obtenidos para D. larteti muestran un patrón en el que los primeros molares superiores e inferiores se encuentran más desgastados que los segundos y éstos más que los terceros (Fig. 5). 

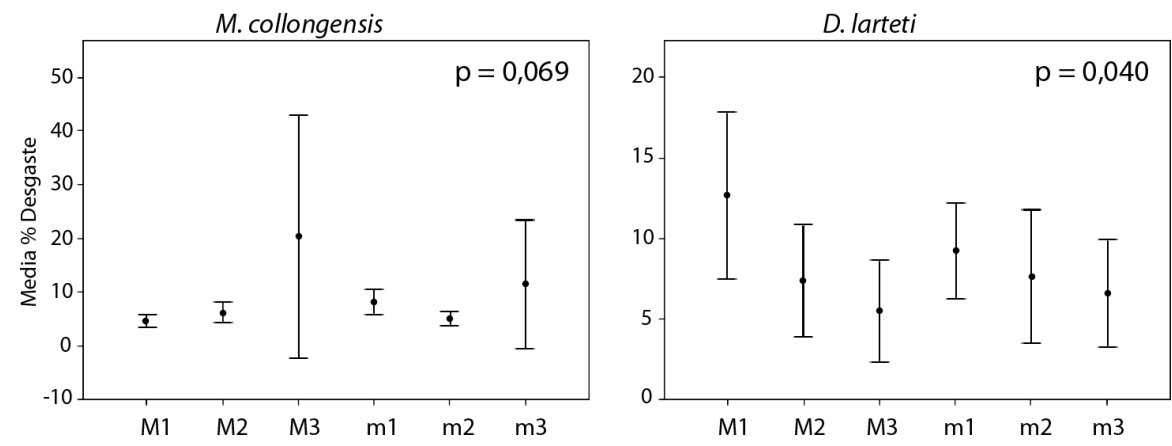

Fig. 5.-Desgaste (media $\pm 95 \%$ del intervalo de confianza) de cada pieza dental de Megacricetodon collongensis (izquierda) y Democricetodon larteti (derecha). Se muestra el valor de significación (p) de la prueba no paramétrica Kruskal-Wallis para las dos especies.

El orden de erupción de los molares en roedores actuales ha sido descrito como un proceso secuencial iniciándose con la erupción de los primeros molares en el día 15 tras el nacimiento y finalizando con la aparición de los terceros molares, que lo hacen aproximadamente a los 18 días de vida (Trout, 1978; Chou et al., 1998; Jernvall \& Thesleff, 2012; Jheon et al., 2013). De esta manera, el lactante posee la dentición completa justo antes del inicio del consumo de materia que puede desgastar la superficie oclusal de los dientes, que se produce entre los 18 y los 30 días de vida (Trout, 1978). Sin embargo, nuestros resultados parecen indicar que el orden de erupción de los molares podría afectar al desgaste de $D$. larteti a pesar de que los individuos todavía sean lactantes. En este caso, el desgaste no se debería al consumo de alimentos duros sino simplemente al roce entre los dientes durante la lactancia. De esta forma los terceros molares resultarían los menos desgastados, dado que son los últimos en desarrollarse.

Por otro lado, este modelo de desgaste también podría explicarse debido al proceso de masticación. Los cricétidos producen movimientos oblicuos en la masticación, haciendo coincidir las cúspides de los molares superiores e inferiores durante la oclusión (Butler, 1980; Lazzari et al., 2008, 2015; Coillot et al., 2013). Sin embargo, el orden en el que se ponen en contacto los molares con el alimento no está descrito, y es posible que los molares M3 y m3 resulten menos desgastados que el resto porque son los últimos en entrar en contacto con el alimento y los últimos encargados de procesarlo.
En cuanto a $M$. collongensis, los resultados no son concluyentes debido al limitado tamaño de muestra de los terceros molares. A pesar de ello, sin tener en cuenta estas piezas, no se observa un modelo claro de desgaste como en $D$. larteti y aparentemente las diferencias de desgaste entre las distintas piezas dentales no son tan acusadas. El desgaste parece ser más homogéneo, lo que podría indicar que en esta especie el uso de las piezas dentales durante la masticación era más uniforme (Fig. 5).

\section{Incidencia de desgaste interespecífica}

La comparación interespecífica de los patrones de desgaste mostró que sólo el patrón del M1 de $M$. collongensis presenta diferencias significativas con el de $D$. larteti, especie en la que aparecen más desgastados, mientras que para el resto de molares las diferencias entre ambas especies no son estadísticamente significativas (Fig. 6).

A pesar de que ambos géneros, Democricetodon y Megacricetodon, presentarían una dieta omnívora dominada por plantas (Coillot et al., 2013), no podemos descartar la posibilidad de que ligeras diferencias dietarias produjeran un desgaste distinto entre las dos especies estudiadas.

Estas diferencias de desgaste entre los M1 también podrían deberse a pequeñas diferencias morfológicas entre ambas especies. La presencia de valles relativamente más estrechos en Democricetodon larteti separando cúspides voluminosas (van der Meulen et al., 2003) favorecerían que la superficie relativa de dentina expuesta con respecto al área 

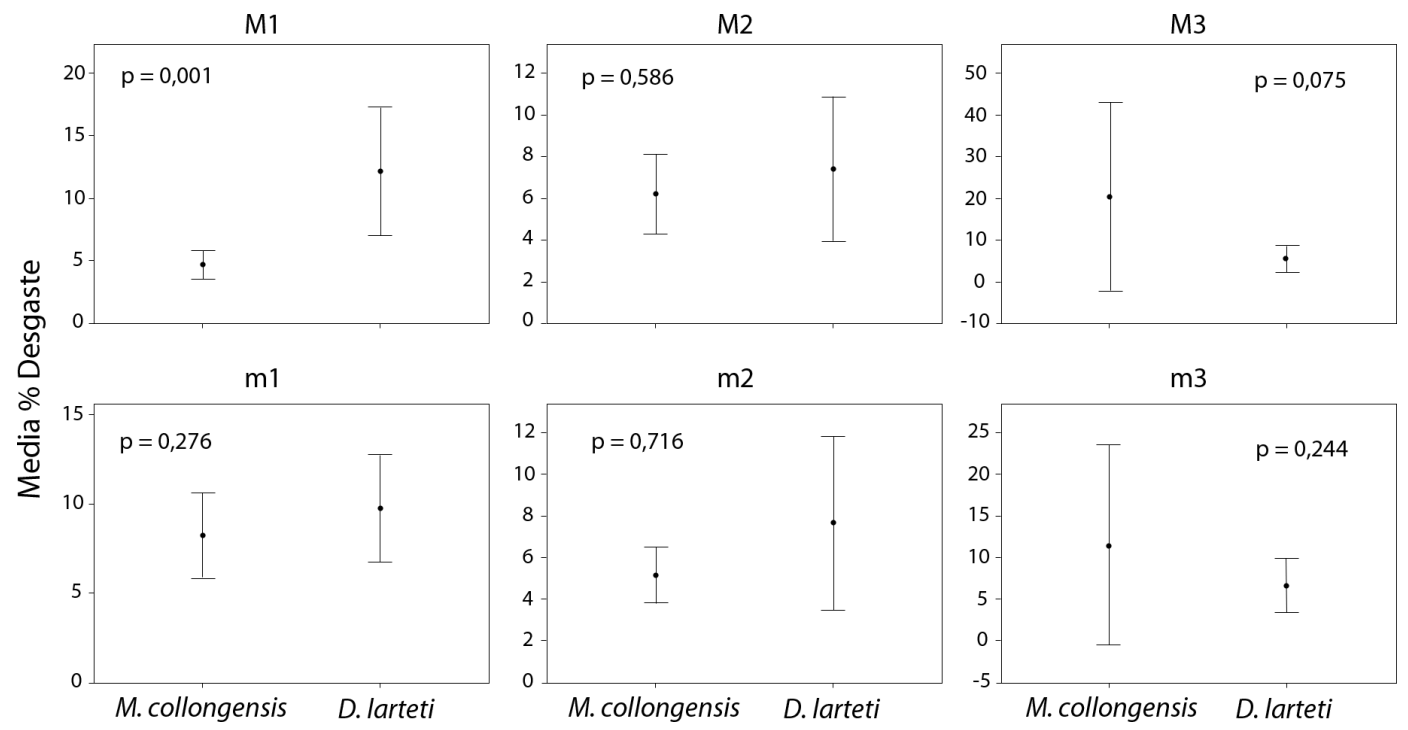

Fig. 6. - Comparación de la media ( $\pm 95 \%$ del intervalo de confianza) de desgaste de los distintos molares entre Megacricetodon collongensis y Democricetodon larteti de Somosaguas-Sur. Se indica el nivel de significación (p) para la comparación de desgaste entre ambas especies a través de una prueba $U$ de Mann-Whitney.

oclusal total se incrementase de manera más rápida en Democricetodon que en Megacricetodon al avanzar el desgaste, por unión de las áreas de dentina de las cúspides adyacentes.

\section{Conclusiones}

Los espectros de desgaste dental de $M$. collongensis y $D$. larteti indican una mortalidad preferencial de individuos juveniles en ambas especies, concordando también con los resultados en otros mamíferos del yacimiento de Somosaguas, y la particular ausencia casi completa de individuos en las categoría de edad más avanzada. Este patrón de mortalidad correspondería a un suceso catastrófico de larga duración, en el que periodos de sequía prolongada produjeron la muerte temprana de los individuos, de manera que no llegaban a alcanzar la senectud.

Nuestros resultados indicaron que todas las piezas dentales producen espectros de desgaste coherentes, por lo que cualquiera de ellas puede ser utilizada en técnicas paleodemográficas mientras no exista un sesgo de muestreo como el detectado en los terceros molares de $M$. collongensis.

$M$. collongensis y D. larteti presentaron diferentes patrones de desgaste en sus hileras dentales. El desgaste diferencial observado en los molares de $D$. larteti, donde los molares más desgastados son los primeros de la hilera dental (M1 y m1) y los menos desgastados son los situados en la zona más interna de la cavidad bucal (M3 y m3), se podría producir de esta manera debido al proceso de masticación, en el que los terceros molares son los últimos que entran en contacto con el alimento o al inicio del desgaste de los molares anteriores ya en el periodo de lactancia, antes de la erupción de los terceros. Por otro lado, en M. collongensis existe un desgaste uniforme de las piezas dentales, por lo que podemos deducir que el uso de los molares en el proceso de masticación sería homogéneo.

Finalmente, diferencias interespecíficas en la dieta o en la morfología oclusal de los molares podrían provocar la diferencia de desgaste observada entre los M1 de ambas especies, donde D. larteti muestra mayores niveles de desgaste que $M$. collongensis a pesar de la supuesta similitud entre las dietas de ambas especies.

\section{AGRADECIMIENTOS}

Los autores desean agradecer el esfuerzo realizado por todos los excavadores y excavadoras que han participado año tras año en las diferentes campañas de excavación y triado realizadas en los yacimientos de Somosaguas; sin ellos las investigaciones asociadas al Proyecto Somosaguas de Paleontología no podrían 
llevarse a cabo. Agradecemos a la Dra. Paloma López Guerrero sus comentarios sobre la figura 2. Las observaciones del Dr. Isaac Casanovas i Vilar y el Dr. Matthijs Freudenthal sobre el manuscrito original han ayudado a mejorar el resultado final. Este trabajo es una contribución del equipo de Paleoclimatología, Macroecología y Macroevolución de Vertebrados (www.pmmv. com.es) de la Universidad Complutense de Madrid, como parte del grupo de investigación UCM 910607 sobre Evolución de Mamíferos y Paleoambientes Continentales Cenozoicos. Este trabajo ha recibido financiación parcial del proyecto CGL201568333-P (MINECO/FEDER, UE) y de la Generalitat de Catalunya (programa CERCA).

\section{Referencias}

Álvarez Sierra, M. A. \& Daams, R. (1987). Pseudotheridomys fejfari, a new species of Eomyidae (Rodentia) from the Ramblian (Lower Miocene) of northern Teruel (Spain). Scripta Geologica, 83: 19-26.

Blanco, F. \& Hernández Fernández, M. (2016). Heterogeneidad lateral en las muestras de roedores del tramo T1 del Mioceno Medio de Somosaguas (Pozuelo de Alarcón, Madrid). Estudios Geológicos, 72(2): e052. http://dx.doi.org/10.3989/egeol.42255.386

Carrasco, A.; Sacristán, S.; Benítez-López, G.; Romero Nieto, D.; Fesharaki, O. \& López-Martínez, N. (2008). Aplicaciones paleoclimáticas y paleoambientales de los estudios mineralógicos al yacimiento de vertebrados miocenos de Somosaguas. In: Palaeontologica Nova (Esteve, J. \& Meléndez, G., Eds.). Publicaciones del Seminario de Paleontología de Zaragoza, Zaragoza, 8: 135-149.

Coillot, T.; Chaimanee, Y.; Charles, C.; Gomes-Rodrigues, H.; Michaux, J.; Tafforeau, P.; Vianey-Liaud, M.; Viriot, L. \& Lazzari, V. (2013). Correlated changes in occlusal pattern and diet in stem murinae during the onset of the radiation of Old World rats and mice. Evolution, 67 (11): 3323-3338. https://doi. org/10.1111/evo.12172

Chou, C.-W.; Lee, P.-F.; Lu, K.-H. \& Yu, H.-T. (1998). A population study of house mice (Mus musculus castaneus) inhabiting rices granaries in Taiwan. Zoological Studies, 37 (3): 201-212.

Daams, R. \& Freudenthal, M. (1981). Aragonian: the stage concept versus Neogene Mammal Zones. Scripta Geologica, 62: 1-17.

Daams, R. \& Freudenthal, M. (1988). Synopsis of the Dutch-Spanish collaboration program in the Aragonian type area, 1975-1986. Scripta Geologica, Special issue, 1: 39-132.

Daams, R.; van der Meulen, A.J.; Álvarez Sierra, M.A.; Peláez-Campomanes, P. \& Krijgsman, W. (1999). Aragonian stratigraphy reconsidered, and a reevaluation of the middle Miocene mammal biochronology in Europe. Earth and Planetary Science Letters, 165 (3-4): 287-294. https://doi.org/10.1016/ S0012-821X(98)00273-8
Díez-Canseco, D.; López-Martínez, N.; Díaz-Molina, M. \& Benito, M.I. (2012). Stream mouth deposits in the paleontological site of Somosaguas, Middle Miocene, Madrid Basin. Spanish Journal of Palaeontology, 27: 93-104.

Domingo, L.; Cuevas-González, J.; Grimes, S.T.; Hernández Fernández, M. \& López-Martínez, N. (2009). Multiproxy reconstruction of the palaeoclimate and palaeoenvironment of the Middle Miocene Somosaguas site (Madrid, Spain) using herbivore dental enamel. Palaeogeography, Palaeoclimatology, Palaeoecology, 272: 53-68. https://doi.org/10.1016/j. palaeo.2008.11.006

Domingo, L.; Koch, P. L.; Grimes, S. T.; Morales, J. \& López-Martínez, N. (2012). Isotopic paleoecology of mammals and the Middle Miocene Cooling event in the Madrid Basin (Spain). Palaeogeography, Palaeoclimatology, Palaeoecology, 339-341: 98-113. https://doi.org/10.1016/j. palaeo.2012.04.026

Domingo, M.S.; Alberdi, M.T.; Azanza, B. \& Morales, J. (2012). Mortality patterns and skeletal physical condition of the carnivorans from the Miocene assemblage of Batallones-1 (Madrid Basin, Spain). Neues Jahrbuch für Geologie und Paläontologie Abhandlungen, 265: 131-145. https://doi. org/10.1127/0077-7749/2012/0251

Domingo, M. S.; Martín-Perea, D.; Domingo, L.; Cantero, E.; Cantalapiedra, J. L.; García Yelo, B. A.; Gómez Cano, A. R.; Alcalde, Gema M.; Fesharaki, O. \& Hernández Fernández, M. (2017). Taphonomy of mammalian fossil bones from the debris-flow deposits of Somosaguas-North (Middle Miocene, Madrid Basin, Spain). Palaeogeography, Palaeoclimatology, Palaeoecology, 465, 103-121.

Fesharaki, O.; Arribas, J. \& López Martínez, N. (2015) Composition of clastic sediments from the Somosaguas area (Middle Miocene, Madrid Basin): insights into provenance and palaeoclimate. Journal of Iberian Geology, 41 (2): 205-222.

Fesharaki, O.; Torices, A.; García Yelo, B.A.; Tejedor Navarro, N.; De la Ossa, L. \& Hernández Fernández, M. (2012). The Somosaguas Palaeontology Project: An envision of Nieves López Martínez for linking science and society. Spanish Journal of Palaeontology, 27 (2): 83-92.

Freudenthal, M. (1966). On the mammalian fauna of the Hipparion-beds in the Calatayud-Teruel Basin (prov. Zaragoza, Spain). Part 1. The genera Cricetodon and Ruscinomys (Rodentia). Proceedings Koninklijke Nederlandse Akademie van Wetenschappen, B, 69 (2): 296-317.

Freudenthal, M.; Martín-Suárez, E. \& Bendala, N. (2002). Estimating age through tooth wear. A pilot study on tooth abrasion in Apodemus (Rodentia, Mammalia). Mammalia, 6: 275-284. https://doi.org/10.1515/ mamm.2002.66.2.275 
Galbany, J.; Romero, A.; Mayo-Alesón, M.; Itsoma, F.; Gamarra, B.; Pérez-Pérez, A.; Willaume, E.; Kappeler, P.M. \& Charpentier, M.J.E. (2014). Agerelated tooth wear differs between forest and savanna primates. PLoS One, 9 (4): e94938. https://doi. org/10.1371/journal.pone.0094938

García Yelo, B.A.; Gómez Cano, A.R.; Cantalapiedra, J.L.; Alcalde, G.M.; Sanisidro, O.; Oliver, A.; Hernández-Ballarín, V.; López-Guerrero, P.; Fraile, S. \& Hernández Fernández, M. (2014). Palaeoenvironmental analysis of the Aragonian (middle Miocene) mammalian faunas from the Madrid Basin based on body-size structure. Journal of Iberian Geology, 40 (1): 129-140. https://doi.org/10.5209/ rev JIGE.2014.v40.n1.44092

Hernández-Ballarín, V.; Oliver, A. \& Peláez-Campomanes, P. (2011). Revisión de las asociaciones de mamíferos del tránsito Aragoniense medio y superior de la cuenca de Madrid. In: Viajando a Mundos Pretéritos (Pérez-García, A.; Gascó, F.; Gasulla J.M. \& Escaso, F., Eds.), Ajuntament de Morella, Morella, 173-182.

Hernández Fernández, M.; Cárdaba, J.A.; CuevasGonzález, J.; Fesharaki, O.; Salesa, M.J.; Corrales, B.; Domingo, L.; Elez, J.; López Guerrero, P.; Sala-Burgos, N.; Morales, J. \& López Martínez, N. (2006). Los yacimientos de vertebrados del Mioceno medio de Somosaguas (Pozuelo de Alarcón, Madrid): implicaciones paleoambientales y paleoclimáticas. Estudios Geológicos, 62: 266-294. https://doi. org/10.3989/egeol.0662126

Hernández Fernández, M.; Salesa, M.J.; Sánchez, I.M. \& Morales, J. (2003). Paleoecología del género Anchitherium von Meyer, 1834 (Equidae, Perissodactyla, Mammalia) en España: evidencias a partir de las faunas de macromamíferos. Coloquios de Paleontología, Volumen Extraordinario, 1: 253-280.

Hohn, A.A.; Scott, M.D.; Wells, R.S.; Sweeney, J.C.; \& Irvine, A.B. (1989). Growth layers in teeth from known-age, free-ranging bottlenose dolphins. Marine Mammal Science, 5 (4): 315-342. https:// doi.org/10.1111/j.1748-7692.1989.tb00346.x

Jernvall, J.; \& Thesleff, I. (2012). Tooth shape formation and tooth renewal: evolving with the same signals. Development, 139 (19): 3487-3497. https://doi. org/10.1242/dev.085084

Jheon, A. H.; Seidel, K.; Biehs, B.; \& Klein, O.D. (2013). From molecules to mastication: the development and evolution of teeth. WIREs Developmental Biology, 2 (2): 165-182. https://doi.org/10.1002/ wdev.63

Kälin, D. (1999). Tribe Cricetini. In: The Miocene Land Mammals of Europe (Rössner, E. \& Heissig, K., Eds.), Verlag Dr. Friedrich Pfeil, München, 373-387.

Krogman, W.M. \& Işcan, M.Y. (1986). The human skeleton in forensic medicine. Charles C. Thomas, Springfield, $551 \mathrm{pp}$.
Laws, R.M. (1952). A new method of age determination for mammals. Nature, 169: 972-973. https://doi. org/10.1038/169972b0

Lazzari, V.; Charles, C.; Tafforeau, P.; Vianey-Liaud, M.; Aguilar, J.P.; Jaeger, J.J.; Michaux, J. \& Viriot, L. (2008). Mosaic convergence of rodent dentitions. PLoS One, 3 (10): e3607. https://doi.org/10.1371/ journal.pone.0003607

Linnaeus, C. (1758). Systema Naturae per regna tria naturae, secundum classis, ordines, genera, species cum characteribus, differentiis, synonymis, locis. Vol. 1. $10^{\mathrm{a}}$ ed. Laurentii Salvii, Stockholm, $824 \mathrm{pp}$.

López-Martínez, N.; Élez, J.; Hernando, J.M.; Luis Cavia, A.; Mazo, A.; Mínguez Gandú, D.; Morales, J.; Polonio, I.; Salesa, M.J. \& Sánchez, I.M. (2000). Los fósiles de vertebrados de Somosaguas (Pozuelo, Madrid). Coloquios de Paleontología, 51: 71-85.

Luis, A. \& Hernando, J.M. (2000). Los microvertebrados del Mioceno Medio de Somosaguas Sur (Pozuelo de Alarcón, Madrid, España). Coloquios de Paleontología, 51: 87-136.

Lyman, R.L. (Eds.) (1994). Vertebrate Taphonomy. Cambridge University Press, Cambridge, 524 pp. https:// doi.org/10.1017/cbo9781139878302

Manzanero, E. \& Hernández Fernández, M. (2014). Patrón de mortalidad en Gomphotherium angustidens del yacimiento de Somosaguas (Pozuelo de Alarcón, Madrid). In: New Insights on Ancient Life (Arreguín Rodríguez, G.J; Colmenar Lallena, J.; Díaz Berenguer, E.; Galán García, J.; Legarda Lisarri, A.; Parrilla Bel, J.; Puértolas Pascual, E. \& Silva Casal, R. Eds.), Prensas de la Universidad de Zaragoza, Zaragoza, 160-162.

Martín Perea, D.M. (2015). New taphonomic inferences on the Middle Miocene vertebrate site of Somosaguas (Pozuelo de Alarcón, Madrid). Trabajo Fin de Master, Universidad Complutense de Madrid, 46 pp.

Mein, P. (1958). Les mammifères de la faune sidérolithique de Vieux-Collonges. Nouvelle archives du Muséum d'histoire naturelle de Lyon, 5: 1-122.

Menéndez, I.; Gómez Cano, A.R. \& Hernández Fernández, M. (2015). Patrón de mortandad de Megacricetodon collongensis (MEIN, 1958) en Somosaguas Sur (Mioceno medio, Pozuelo de Alarcón, Madrid). In: Current Trends in Paleontology and Evolution (Domingo, L.; Domingo, M. S.; Fesharaki, O.; García Yelo, B.; Gómez Cano, A.R.; Hernández-Ballarín, V.; Hontecillas, D.; Cantalapiedra, J. L.; López Guerrero, P.; Oliver, A.; Pelegrín, J.; Pérez de los Ríos, M.; Ríos, M.; Sanisidro O. \& Valenciano, A., Eds.), EJIP, Cercedilla, 188-190.

Menéndez, I.; Gómez Cano, A.R. \& Hernández Fernández, M. (2016). A new mortality profile in fossil sites. In: New perspectives on the Evolution of Phanerozoic Biotas and Ecosystems Conference proceedings. (Manzanares, E.; Ferrón, H.G.; Suñer, M.; Holgado, B.; Crespo, V.D.; Mansino, 
S.; Fagoaga, A.; Marquina, R.; García-Sanz, I.; Martínez-Pérez, C.; Joanes-Rosés, M.; CascalesMiñana, B. \& Marin-Monfort, M.D. Eds.). Ayuntamiento de Alpuente, 108.

Mínguez Gandú, D. (2000). Marco estratigráfico y sedimentológico de los yacimientos paleontológicos miocenos de Somosaguas (Madrid, España). Coloquios de Paleontología, 51: 183-196.

Morris, P. (1972). A review of mammalian age determination methods. Mammal review, 2 (3): 69-104. https://doi.org/10.1111/j.1365-2907.1972.tb00160.x

Pallas, P.S. (1771-1776). Reise durch verschiedene Provinzen des Russischen Reichs. J.G. Fleischer, St. Petersbourg, 3 vols.

Perales, R.; Serrano, H.; García Yelo, B.A. \& Hernández Fernández, M. (2009). Inferencias paleoambientales del Mioceno medio de Somosaguas (Pozuelo de Alarcón, Madrid) basadas en la estructura de tamaños corporales de su fauna de mamíferos. Paleolusitana, 1: 317-325.

Polonio, I. \& López-Martínez, N. (2000). Análisis tafonómico de los yacimientos de Somosaguas (Mioceno Medio, Madrid). Coloquios de Paleontología, 51: 235-266.

Schaub, S. (1925). Die hamsterartigen nagetiere des Tertiärs und ihre lebenden Verwandten. Abdhalungen der Schweizerischen Paläonologischen Gesellschaft, 45: $1-114$.

SPSS. (2006). Statistical Package for the Social Sciences. Versión 15.0. SPSS Inc, Chicago.

Torroba, J.; Gómez de la Peña, L.; Gómez Cano, A.R.; López-Guerrero, P. \& Hernández Fernández, M. (2010). Estudio de los patrones de mortandad de Democricetodon larteti (Schaub, 1925) en el yacimiento mioceno de Somosaguas (Pozuelo de Alarcón, Madrid). Cidaris, 30: 325-328.

Trout, R.C. (1978). A review of studies on populations of wild harvest mice añadir cursiva (Pallas). Mammal Review, 8 (4): 143-158. https://doi. org/10.1111/j.1365-2907.1978.tb00224.x

Ubelaker, D.H. (1987). Estimating age at death from immature human skeletons: an overview. Journal of Forensic sciences, 32 (5): 1254-1263. https://doi. org/10.1520/JFS11176J

Van der Meulen, A.J.; García-Paredes, I.; Álvarez-Sierra, M.A.; van den Hoek Ostende, L.W.; Hordijk, K.; Oliver, A. \& Peláez-Campomanes, P. (2012). Updated Aragonian biostratigraphy: small mammal distribution and its implications for the Miocene European chronology. Geologica Acta, 10 (2): 159-179.

Van der Meulen, A.J.; García-Paredes, I.; Álvarez-Sierra, M.A.; van den Hoek Ostende, L.W.; Hordijk, K.; Oliver, A.; López-Guerrero, P.; Hernández-Ballarín, V. \& Peláez-Campomanes, P. (2011). Biostratigraphy or Biochronology? Lessons from the Early and Middle Miocene small Mammal events in Europe. Geobios, 44: 309-321. https://doi.org/10.1016/j. geobios.2010.11.004

Van der Meulen A.J.; Peláez-Campomanes, P. \& Daams, R. (2003). Revisión de los cricétidos de talla media del Mioceno del área Daroca-Villafeliche en la cuenca de Calatayud-Teruel (Zaragoza, España). Coloquios de Paleontología, Volumen Extraordinario, 1: 253-280.

White T.D. (1991). Human osteology. Academic Press, Inc. New York, 555 pp. 